Supplementary Information for

\title{
Substrate-Sensitive Graphene Oxidation
}

Zhuhua Zhang, Jun Yin, Xiaofei Liu, Jidong Li, Jiahuan Zhang and Wanlin Guo

State Key Laboratory of Mechanics and Control of Mechanical Structures, Key Laboratory for Intelligent Nano Materials and Devices of the Ministry of Education, and Institute of Nanoscience, Nanjing University of Aeronautics and Astronautics, Nanjing 210016, China

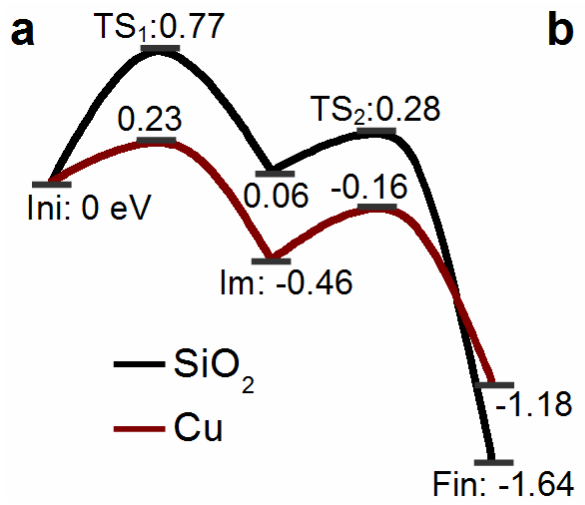

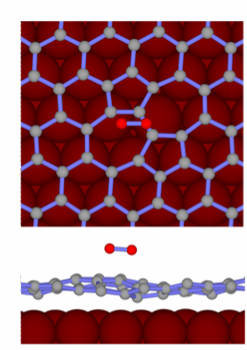

Ini

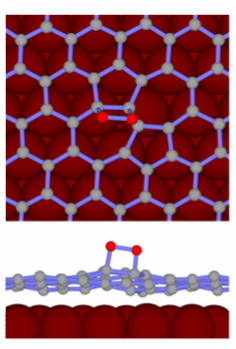

Im

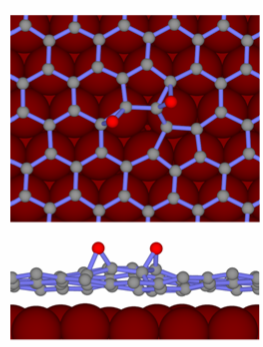

Fin

$\mathrm{CuOCOO}$

Figure S1. Oxygen chemisorption and dynamics at defects in graphene. (a) Calculated minimum energy paths from the physisorption state (Ini), to the chemisorption state (Im) and finally to the dissociated epoxy groups (Fin) of an $\mathrm{O}_{2}$ at a Stone-Wales defect in graphene on $\mathrm{SiO}_{2}$ (black) and $\mathrm{Cu}$ (wine) substrates. (b) Physisorption (Ini), chemisorption (Im) and dissociated (Fin) configurations of $\mathrm{O}_{2}$ at a Stone-Wales defect in the $\mathrm{Cu}$-supported graphene. 


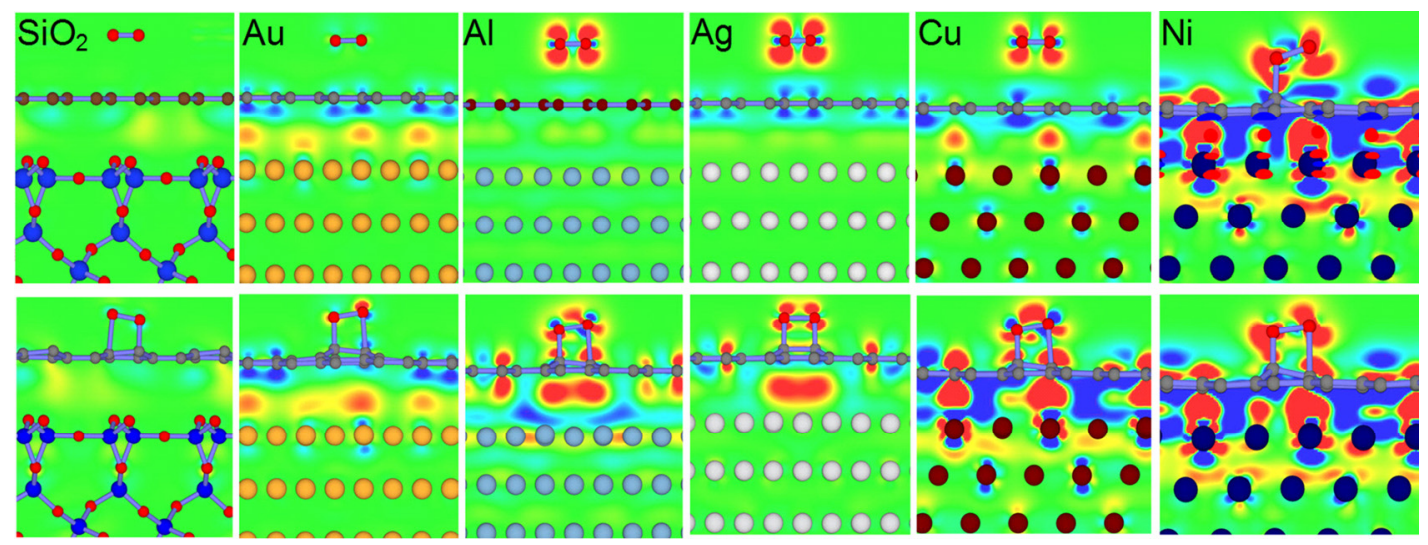

Figure S2. Charge transfer between graphene and substrates. The O-crossed slices of charge redistribution between the substrate and graphene plus $\mathrm{O}_{2}$. Blue and red colors represent charge depletion and accumulation regions, respectively, with the same isosurface value. The charge difference density is calculated by $\Delta \rho=\rho_{\mathrm{sys}}-\rho_{\mathrm{sub}}-\rho_{\mathrm{gr}}-\rho_{\mathrm{O} 2}$, where $\rho_{\text {sys }}, \rho_{\text {sub }}, \rho_{\text {gr }}$, and $\rho_{\mathrm{O} 2}$ are the total charge densities of the system, substrate, graphene and $\mathrm{O}_{2}$ molecule, respectively, calculated within the same simulation box.

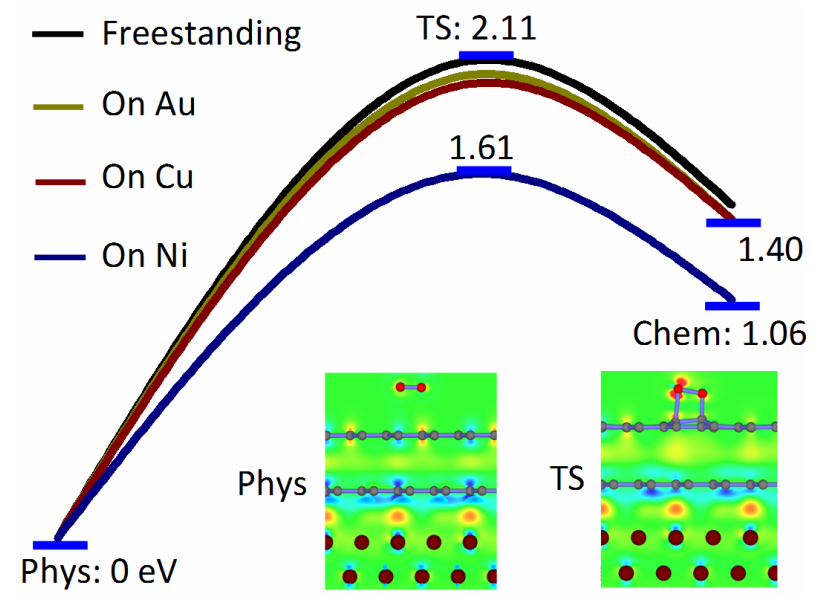

Figure S3. Oxidation of bilayer graphene on substrates. Calculated minimum energy path between the physorption and chemisorption states of a $\mathrm{O}_{2}$ on a freestanding bilayer graphene as well as $\mathrm{Au}, \mathrm{Cu}$ and $\mathrm{Ni}$ supported bilayer graphene. Side views of the physisorption (Phys) and transition state (TS) configurations on the $\mathrm{Cu}$ substrate as well as the charge redistribution between $\mathrm{Cu}$, graphene and $\mathrm{O}_{2}$. Red and blue colors represent the charge accumulation and depletion regions, respectively. 

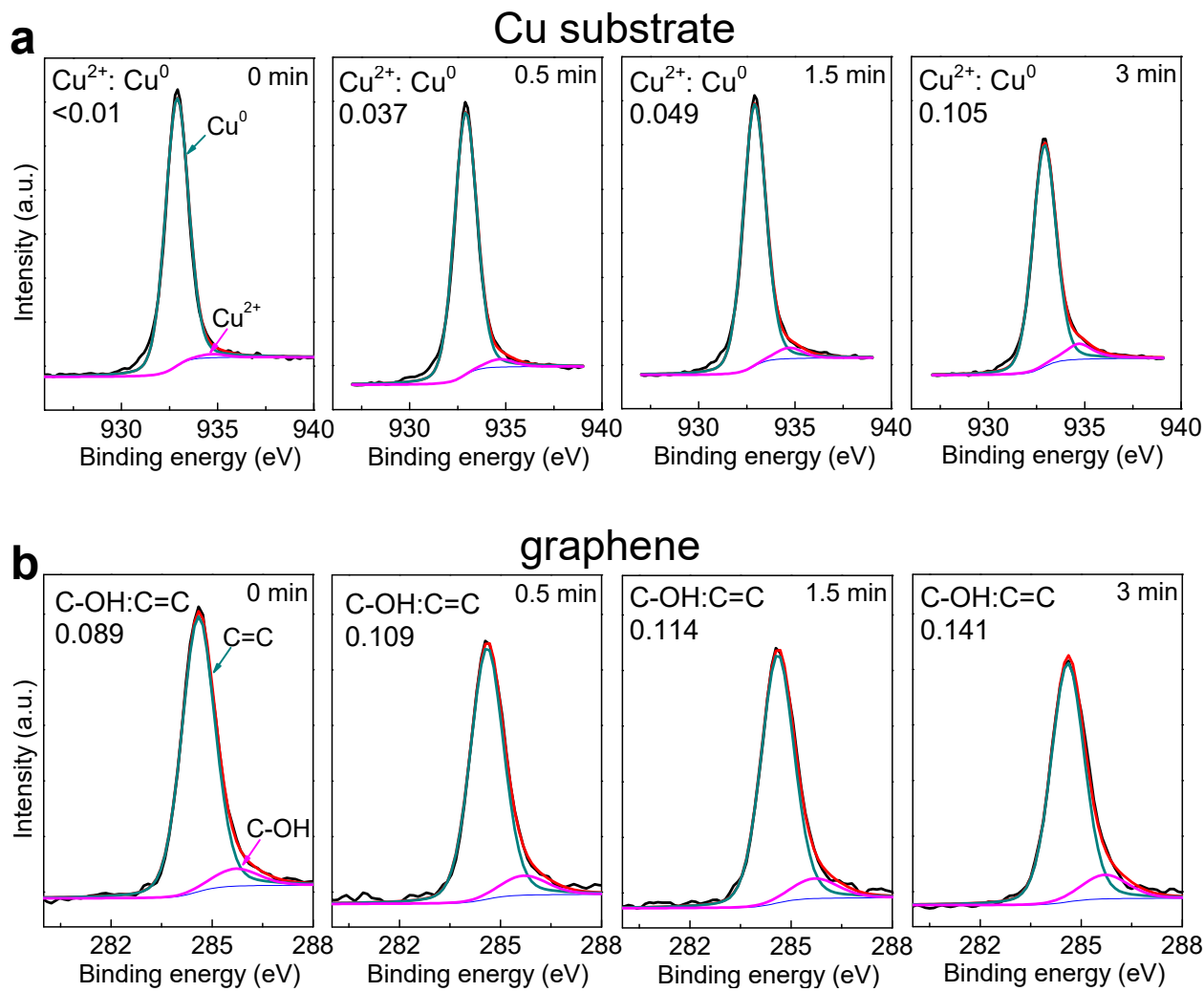

graphene
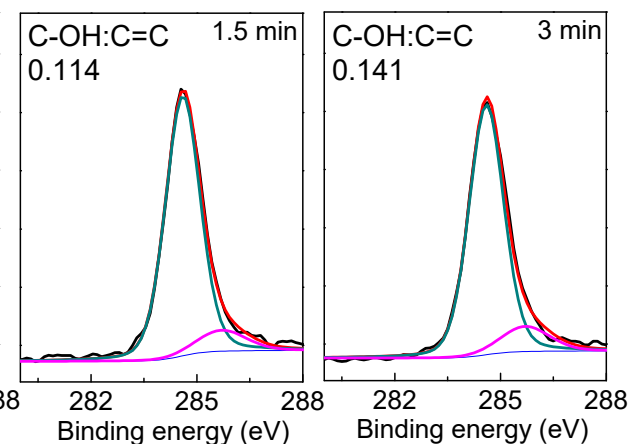

Figure S4. XPS characterization of oxidized graphene on substrates. (a) Evolution of $\mathrm{X}$-ray photoelectron spectroscopy (XPS) spectra of the $\mathrm{Cu}$ substrate by exposing the $\mathrm{Cu}$ gr in air at $270{ }^{\circ} \mathrm{C}$. Peak-differentiated spectra for the $\mathrm{Cu}^{2+}$ and $\mathrm{Cu}$ are shown in pink and dark cyan, respectively. (b) XPS spectra of the supported graphene. Peak-differentiated spectra of the $\mathrm{C}=\mathrm{C}$ double bond $(284.5 \mathrm{eV})$ and $\mathrm{C}-\mathrm{OH}$ hydroxyl $(286.7 \mathrm{eV})$ are shown in pink and dark cyan, respectively. The ratio of $\mathrm{Cu}^{2+}$ to $\mathrm{Cu}^{0}$ in the substrate and that of $\mathrm{C}$ $\mathrm{OH}: \mathrm{C}-\mathrm{C}$ in the graphene are provided in each figure. Note that all $\mathrm{C}-\mathrm{O}-\mathrm{C}$ and $\mathrm{C}=\mathrm{O}$ groups may have been converted to the hydroxyl groups during the sample delivery and XPS measurements.

The small oxygen concentration in graphene before oxidation is due to the contamination and it does not undergo notable change until the oxidation testing is performed. 


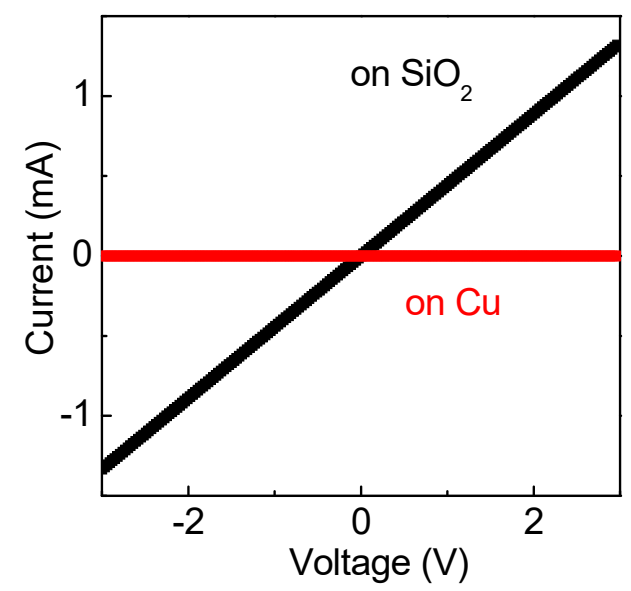

Figure S5. I-V curves of oxidized graphene on $\mathrm{SiO}_{2}$ and $\mathbf{C u}$. Both the samples are exposed in dry air for $30 \mathrm{~min}$ at a temperature of $270{ }^{\circ} \mathrm{C}$. The oxidized sample on $\mathrm{Cu}$ was transferred to a $\mathrm{SiO}_{2} / \mathrm{Si}$ substrate for electric measurement.
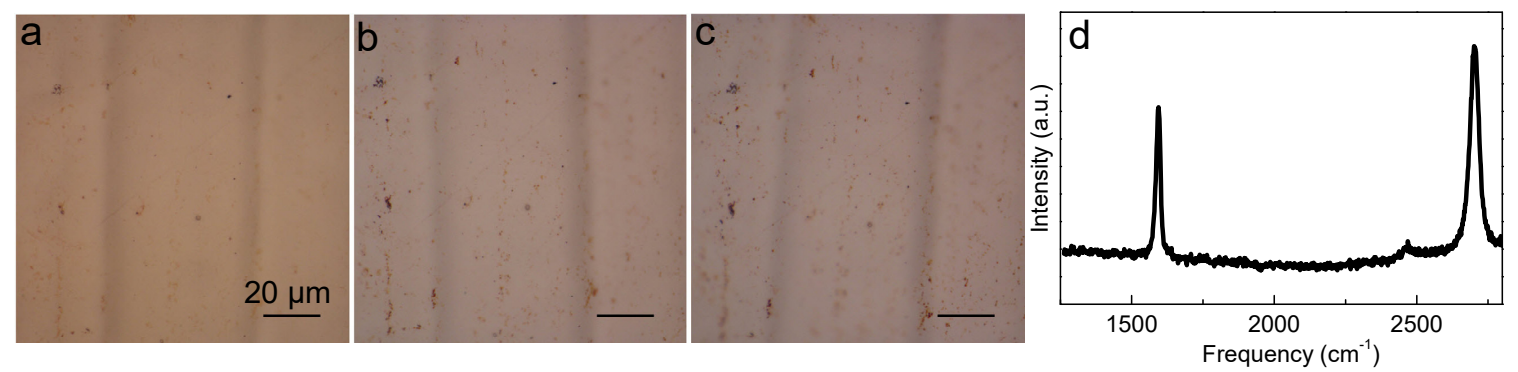

Figure S6. Experimental characterization of oxidation of bilayered graphene on $\mathbf{C u}$. (a-c) Optical image of a bilayered graphene sample on $\mathrm{Cu}$, which is exposed in dry air at $270{ }^{\circ} \mathrm{C}$ for (a) $0 \mathrm{~min}$, (b) $3 \mathrm{~min}$ and (c) $6 \mathrm{~min}$. (d) Raman spectra of the bilayered graphene sample on $\mathrm{Cu}$ exposed in dry air for $6 \mathrm{~min}$. 


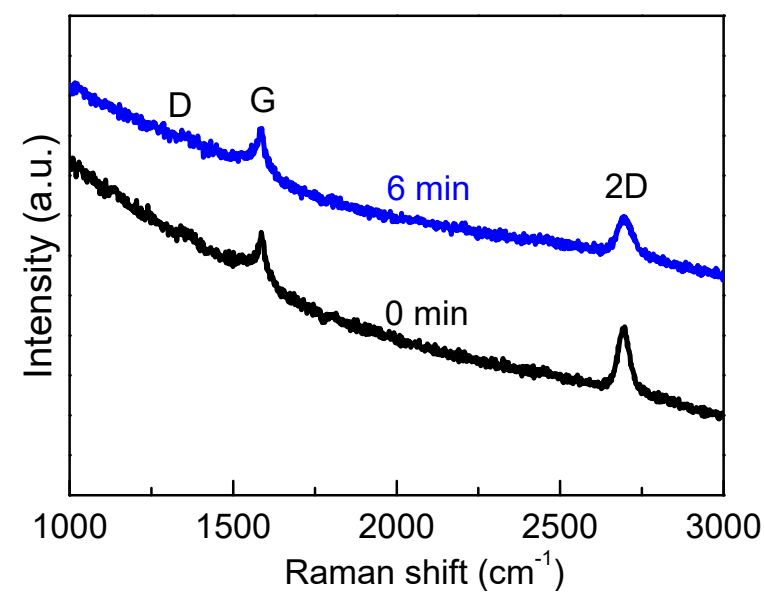

Figure S7. Raman spectra of the graphene samples on Au exposed in dry air for $0 \mathrm{~min}$ (black) and $6 \mathrm{~min}$ (blue) at $270{ }^{\circ} \mathrm{C}$. It is shown that the graphene on Au show no measurable Raman signal of defects upon exposure, indicating its high oxidation resistance and consistent with our theoretical results.

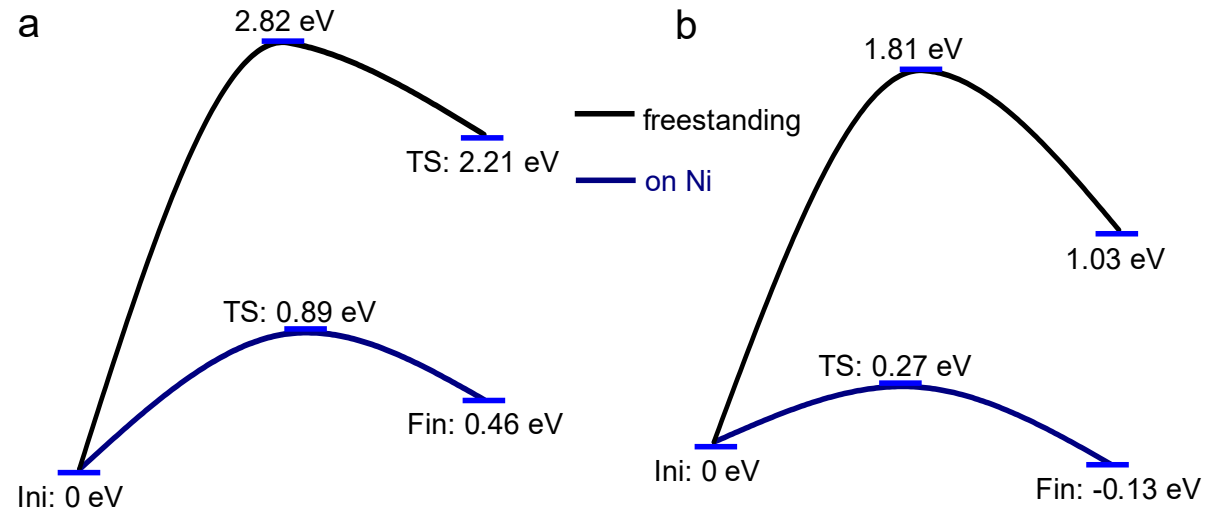

Figure S8. Testing our models using different lattice parameter and exchange correlation potential. (a) Calculated minimum energy paths between the physorption and chemisorption states of an $\mathrm{O}_{2}$ on freestanding and $\mathrm{Ni}$-supported graphene using generalized gradient approximation. (b) Calculated minimum energy paths on freestanding and Ni-supported single-layered graphene using local spin density approximation, with the lattice of the system adopted as that of the Ni substrate.

Even though the generalized gradient approximation significantly overestimates the graphene-metal distance, the substrate-dependent oxidation dynamics of graphene remains qualitatively unchanged. 